\title{
The effectiveness of education using the health belief model in preventing osteoporosis among female students
}

\author{
H. Sanaeinasab, ${ }^{1}$ R. Tavakoli, ${ }^{1}$. Karimizarchi, ${ }^{2}$ Z. Haji Amini, ${ }^{3}$ A. Farokhian ${ }^{7}$ and F. Rahmati Najarkolaei ${ }^{4}$
}

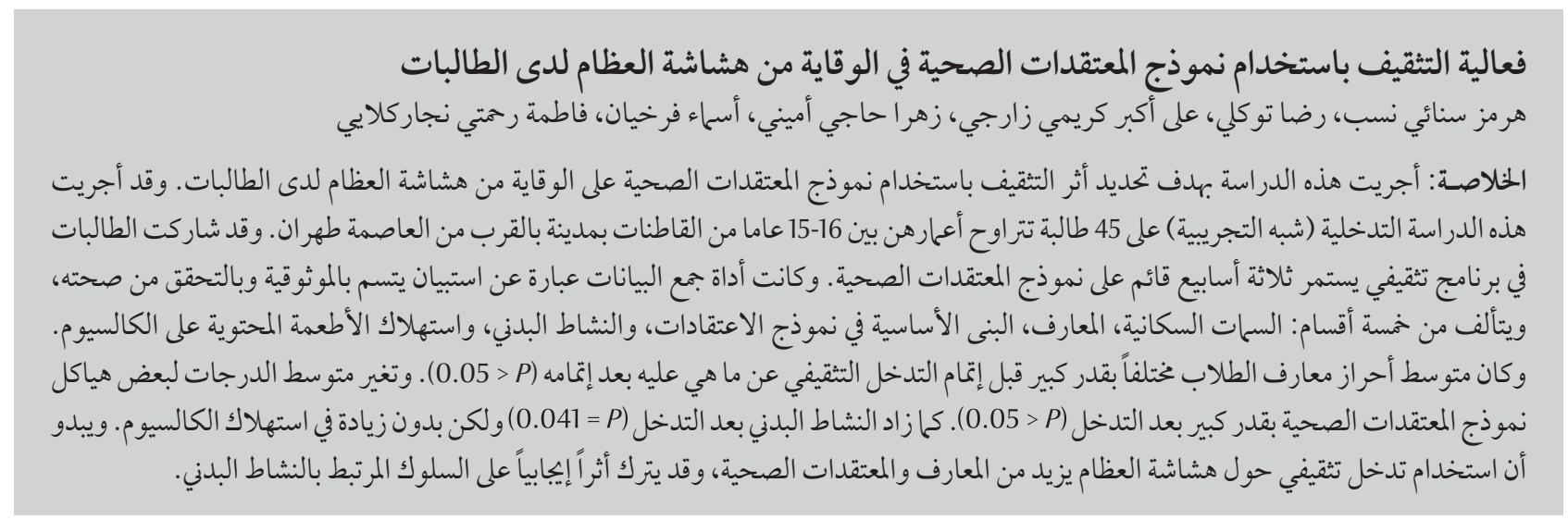

ABSTRACT This study was conducted to determine the impact of education using the Health Belief Model on preventing osteoporosis among female students. This interventional study (quasi-experimental) was performed on 45 female students aged 15-16 years old who resided in a town near Tehran. The females participated in a threeweek educational programme based on the Health Belief Model. The data collection instrument was a validated and reliable questionnaire in five sections: demographics, knowledge, Health Belief Model constructs, physical activity and consumption of foods containing calcium. The mean scores of students' knowledge were significantly different before and after the educational intervention $(P<0.05)$. The mean scores of some Health Belief Model structures changed significantly after the intervention $(P<0.05)$. Also post-intervention, physical activity increased $(P=0.041)$ but calcium intake did not. The use of an educational intervention on osteoporosis seems to improve knowledge and health beliefs and may positively impact physical activity-related behaviour.

Efficacité d'un programme éducatif fondé sur le modèle des croyances relatives à la santé pour prévenir l'ostéoporose chez des lycéennes

RÉSUMÉ La présente étude a été menée pour déterminer l'impact d'un programme éducatif utilisant le modèle des croyances relatives à la santé sur la prévention del'ostéoporose chez des lycéennes. Cette étude interventionnelle (quasiexpérimentale) a été menée auprès de 45 lycéennes, âgées de 15 ou 16 ans, résidant dans une ville proche de Téhéran. Les jeunes filles ont participé à un programme éducatif de trois semaines, fondé sur le modèle des croyances relatives à la santé. Les données ont été recueillies à l'aide d'un questionnaire validé et fiable, comportant cinq sections : les caractéristiques démographiques; les connaissances; les concepts du modèle des croyances relatives à la santé ; l'activité physique; et la consommation d'aliments riches en calcium. S'agissant des connaissances, la différence entre les scores moyens des lycéennes avant et après l'intervention éducative était significative $(p<0,05)$, de même que pour les concepts du modèle des croyances relatives à la santé $(p<0,05)$. Le niveau d'activité physique était supérieur après l'intervention, mais l'apport en calcium n'avait pas augmenté $(p=0,041)$. L'utilisation d'une intervention éducative sur l'ostéoporose semble améliorer les connaissances ainsi que les croyances relatives à la santé, et peut avoir des effets positifs sur le comportement lié à l'activité physique.

${ }^{7}$ Health Education Department, School of Health, Baqiyatallah University of Medical Sciences, Tehran, Islamic Republic of Iran. ${ }^{2}$ Epidemiology and Biostatistics Department, School of Health, Baqiyatallah University of Medical Sciences, Tehran, Islamic Republic of Iran. ${ }^{3}$ Faculty of Nursing, Baqiyatallah University of Medical Sciences, Tehran, Islamic Republic of Iran. ${ }^{4}$ Health Research Centre, Baqiyatallah University of Medical Sciences, Tehran, Islamic Republic of Iran (Correspondence to F. Rahmati Najarkolaei:fatemeh_rahmaty@yahoo.com).

Received: 20/05/12; accepted: 13/08/12 


\section{Introduction}

Osteoporosis is a global health issue along with heart disease, stroke, diabetes and cancer and takes up many financial resources for prevention and treatment $[1,2]$. It affects more than 75 million people worldwide and, according to the US Department of Health and Human Services, will affect more than 10 million women by 2020 if efforts to prevent it are ineffective [3].

There is a significant difference in the prevalence of osteoporosis among different countries. Among the countries of the WHO Eastern Mediterranean Region, osteoporosis is a health priority. The incidence of osteoporosis and osteopenia among Iranian women and men was found to be $32.4 \%$ and $9.4 \%$ respectively [4]. In a study by Salehi et al. (2009) on Iranian women under 50 years old, $49.6 \%$ of the female population were found to have low bone mass [5]. In the general population, the levels of osteoporosis and osteopenia in the Islamic Republic of Iran were $22.2 \%$ and $59.9 \%$, respectively. The level of osteoporosis is less than that in Pakistan with 55\% [6], Turkey with 27\%-33.3\% [7], Morocco with $31 \%$, Egypt with 28.4\%, Bahrain with $27.1 \%$, Kenya with $24.5 \%$, Saudi Arabia with 23\%-24\% and also less than United Arab Emirates with 2.5\% osteoporosis [8].

Osteoporosis prevalence is higher in females than males [9]. Most bone mass density and content in girls is formed between 11 and 14 years of age [10]. Therefore, more attention should be paid to this age group. Maximizing bone mass along with lifestyle factors such as calcium intake and physical activity are considered as modifiable factors in the prevention of osteoporosis [11].

Curative and preventive schedules for osteoporosis by health education and promotion programmes are effective and cheap ways of increasing knowledge and skills needed to establish behavioural changes like increasing calcium intake and physical activity, two of the known variable factors preventing osteoporosis [12].

The Health Belief Model was first introduced in the early 1950s and updated in the 1980s. It is one of the theoretical models that explain factors influencing healthy behaviour [13]. It is also the most widely applied theoretical framework for evaluating osteoporosis health beliefs and behaviour [14].

Several studies have already been conducted on osteoporosis-preventing interventions using the Health Belief Model in most of which health beliefs and knowledge improved after the intervention, such as the studies by Chan et al. (2007) [15] on young adults, Turner et al. (2004) [16] on middle-aged women and Hazavehei et al. (2007) on Iranian secondary-school girls [17].

With the recent increase in women's life expectancy, the incidence of this disease will grow, and girls are a key target group in preventive intervention. This study was conducted with the purpose of showing the effects of Health Belief Model-based education on the promotion of osteoporosispreventing behaviour among female students.

\section{Methods}

\section{Study design and sample}

In this quasi-experimental pre- and post-intervention study, 48 female students were recruited (three dropped out) from two high schools in municipality district 1 of Tehran through cluster random sampling. District 1 is located in the north of Tehran, and Mahallati section is in the centre of district 1 . The local ethics review committee of Baqiyatallah University of Medical Sciences approved the study protocol. All participants gave written informed consent before participation.

\section{Inclusion criteria}

Participants were 15-16 years old, had no risk factors for osteoporosis and complications of this condition, were willing to participate in study and had no limitations in physical movement and diet.

\section{Study instruments and measures}

The method of data collection was by self-administrated questionnaire containing five parts and 74 questions comprising the following:

- personal information (9 questions)

- knowledge (16 questions)

- Health Belief Model constructs (43 questions)

- physical activity (6 questions)

- and one multiple choice checklist on calcium intake.

\section{Validity}

For establishment and formalization of content validity, the questionnaire was given to 15 experts in the fields of health education, social medicine, rheumatology, nutrition, nursing and epidemiology, and their critical comments were taken into account.

\section{Reliability}

The questionnaire was given to $16 \mathrm{stu}$ dents who had the same backgrounds. The forms were filled out, and Cronbach's coefficient for each section was calculated at more than 0.7 , which is acceptable.

Eight questions were edited in the knowledge section and two were deleted. In the Health Belief Model constructs section, five questions were edited; and seven questions investigating barriers to physical activity were deleted.

\section{Scoring}

In the knowledge part of the questionnaire, every correct and wrong answer was given 1 and 0 points respectively. 
Therefore, the total score appointed for this part was between 0 and 48, and the mean score was divided into three categories: weak $(<50 \%)$, moderate (50\%-75\%) and good (> 75\%).

For analysing the Health Belief Model constructs section of the questionnaire, a five-point Likert scale was used. The mean score for different parts was categorized as weak $(<50 \%)$, moderate $(50 \%-75 \%)$ or good $(>75 \%)$.

In the physical activity part, the physical activity of the teenage girls was calculated by adding the amount of weight-sustaining activities, endurance activities plus walking in minutes in the period of a week. This was then converted to hours per week; and the average amount of physical activity was calculated and categorized as low ( $<5$ hours per week), medium (5-7.5 hours per week) or high ( $>7.5$ hours per week) [18].

A table with a list of foods containing calcium was presented to the participants; since the aim of the study was to affect calcium intake behaviour and beliefs and physical activity, daily and weekly amount of consumption of foods containing calcium, such as dairy produce, were recorded, and the amount of intake calcium per 100 grams of food intake was calculated based on the nutrition combination table and the participants' self-reports.

To calculate the average calcium intake of teenage girls from the calcium intake questionnaire, reliable academic books and charts that showed the amount of calcium in each food type per gram were used. Then, to calculate the mean of the individual daily calcium intake the amount of calcium in each food was multiplied by its consumption frequency [19].

Afterwards, the obtained value was multiplied by the number of intakes. Therefore, the average calcium intake from food sources in addition to calcium supplement pills and syrups was calculated.
A week before and one month after the intervention, the questionnaires were filled out by the students in the presence of the investigator.

\section{Intervention}

Three group sessions of 60 minutes per week.

\section{Teaching method}

Lecture, question and answer, brainstorming, group discussion with pamphlets about the role of nutrition and physical activity in disease prevention and a booklet on osteoporosis.

\section{The content of educational sessions}

Definition, statistics, costs and sideeffects of osteoporosis; risk factors for the disease; symptoms, diagnosis and treatment; emphasis on preventive factors, especially the role of foods containing calcium and physical activity; calculation of daily calcium intake; and exercises promoting bone density.

\section{Analysis}

Data were analysed with SPSS, version 15 , and the paired-sample $t$-test was used to compare mean scores before and after the intervention. Pearson's correlation was used to analyse the relationship between variables.

\section{Results}

In this study, 48 female high school students aged 15-16 participated in the educational intervention. Three of them left the study during the intervention or after the test (Table 1). Table 1 presents the subjects' demographic information. Three subjects were excluded from the study as they had missed more than one session and were reluctant to contribute in the intervention and post-testing.

With respect to knowledge the results showed that before the educational intervention the level of knowledge of only one of the subjects was good (2.2\%), that of 19 was medium
(39.6\%) and that of 28 was low (58.2\%) while afterwards knowledge of 15 of the subjects was medium (33.3\%) and that of 30 subjects was good (66.7\%); and no-one with a low level was reported. According to Table 2, the average knowledge scores about osteoporosis in both tests (pre- and post-test) were 22.91 (SD 4.3) and 37.91 (SD 5.01), and the results of the paired $t$-test indicated a significant difference between them before and after the education $(P$ $<0.001$ ).

Also the results showed that, before the intervention the score of perceived susceptibility of seven subjects was evaluated as good (15.6\%), 24 subjects as medium $(53.3 \%)$ and 14 subjects as poor (31.1\%); however, after the intervention these results were 20 subjects (45.5\%), 21 subjects $(47.7 \%)$ and 3 subjects (4.8\%), respectively (one subject was missing). According to Table 2, the paired $t$-test conveyed a significant difference between the scores of perceived susceptibility, and the mean before the intervention was 17.46 (SD 2.57) and after $19.73($ SD 2.65) $(P<$ 0.001).

Before the intervention good knowledge of the severity of osteoporosis was perceived by nine subjects (20\%), medium by 25 subjects (55.6\%) and poor by 11 subjects (24.4\%); this changed after the intervention to 16 subjects (35.6\%), 20 subjects (44.4\%) and 5 subjects (11.1\%), respectively. The mean of the perceived severity scores in pre- and post-intervention were 21.95 (SD 4.28) and 23.20 (SD 4.63), respectively, which showed no significant difference according to the paired $t$-test. $(P=0.076)$.

On perceived benefits of calcium intake before the intervention, 33 of the subjects had a good score (73.3\%), 12 subjects a middle score (26.7\%) and none of the samples had a poor understanding of the issue; however after the intervention these scores reached 35 subjects $(83.3 \%)$ and 7 subjects (16.7\%), respectively (three 


\begin{tabular}{|c|c|c|}
\hline Variable & No. (total) & $\%$ \\
\hline \multicolumn{3}{|l|}{ Field of education } \\
\hline Human sciences & 12 & 25 \\
\hline Experimental science & 12 & 25 \\
\hline Mathematics & 24 & 50 \\
\hline \multicolumn{3}{|l|}{ Job offather } \\
\hline Employee & 24 & 50 \\
\hline Private & 10 & 20.8 \\
\hline Retired & 14 & 29.2 \\
\hline \multicolumn{3}{|l|}{ Educational level of father ${ }^{a}$} \\
\hline Secondary & 1 & 2.1 \\
\hline High school & 1 & 2.1 \\
\hline Diploma & 11 & 22.9 \\
\hline Academic & 35 & 72.9 \\
\hline \multicolumn{3}{|l|}{ Educational level of mother } \\
\hline Illiterate & 1 & 2.1 \\
\hline Primary & 0 & 10.3 \\
\hline Secondary & 5 & 16.7 \\
\hline Incomplete high school education & 8 & 54.2 \\
\hline High school diploma & 26 & 54.7 \\
\hline University degree & 8 & 16.7 \\
\hline
\end{tabular}

Three students were excluded from the study.

${ }^{a}$ No fathers had less than secondary education.

students were missing). As shown in Table 2, the paired $t$-test did not suggest any significant difference among perceived benefits scores, the mean being 9.33 (SD 1) before the intervention and after 9.38 (SD 0.98) $(P=0.75)$.

Before the intervention, the perceived benefits of physical activity of 27 subjects was good (57.4\%), of 18 subjects
(38.3\%) was medium and of only two subjects was poor (4.3\%) while these numbers after the intervention were 31 subjects (72.1\%), 11 subjects (25.6\%) and 1 subject (2.3\%), respectively. As shown in Table 2, the mean of perceived benefits of physical activity before the intervention was 13.04 (SD 1.87) and after the intervention was 13.61 (SD 1.65); and the paired $t$-test showed this difference as insignificant $(P=0.058)$.

Before the intervention, 30 of the subjects had low knowledge of barriers of calcium intake (65.2\%), 15 subjects had a middle score (32.6\%) and only one subject had a high score (2.2\%) whereas these scores after the intervention were 30 subjects $(68.2 \%)$ and 14 subjects (31.8\%) respectively; there was no one with high amount of barriers. As seen in Table 2, the mean scores of perceived barriers to calcium intake pre- and post-intervention were 8.66 (SD 2.78) and 8.19 (SD 2.53); the paired $t$-test presented this difference as insignificant $(P=0.187)$

Prior to the education, 13 subjects had low knowledge of barriers of physical activity (28.3\%), 25 subjects with middle (54.3\%) and eight subjects with high knowledge of barriers (17.4\%); however, these scores after the intervention were 20 subjects (48.8\%), 19 people (46.3\%), and 2 subjects (4.9\%), respectively. As seen in Table 2, the means of the scores of perceived barriers of physical activity in pre- and postintervention were 27.82 (SD 7.48) and 23.30 (SD 7.31), respectively, and the paired $t$-test indicated a significant difference between the means pre- and post-intervention $(P<0.001)$.

Self-efficacy of calcium intake of the participants before the intervention was as 18 subjects at good level

\begin{tabular}{lcccc}
\hline Table 2 Difference in the participants' knowledge and health belief & model constructs before and after the intervention \\
\hline Variable & $\begin{array}{c}\text { Before intervention } \\
\text { Mean (SD) }\end{array}$ & $\begin{array}{c}\text { After intervention } \\
\text { Mean (SD) }\end{array}$ & \multicolumn{1}{c}{$\boldsymbol{P}$-value } & \multicolumn{1}{c}{-value } \\
Knowledge & $22.91(4.30)$ & $37.91(5.01)$ & $<0.001$ & 13.997 \\
Perceived susceptibility & $17.46(2.57)$ & $19.73(2.65)$ & $<0.001$ & 5.929 \\
Perceived severity & $21.95(4.28)$ & $23.20(4.63)$ & 0.076 & 1.825 \\
Perceived benefits of calcium & $9.33(1.00)$ & $9.38(0.98)$ & 0.321 & 0.750 \\
Perceived benefits of physical activity & $13.04(1.87)$ & $13.61(1.65)$ & 0.058 & 1.947 \\
Perceived barriers of calcium & $8.66(2.78)$ & $8.19(2.53)$ & 0.187 & 1.343 \\
Perceived barriers of physical activity & $27.82(7.48)$ & $23.30(7.31)$ & $<0.001$ & 5.753 \\
Self-efficacy of calcium & $18.85(5.43)$ & $21.34(4.85)$ & $<0.001$ & 4.44 \\
Self-efficacy of physical activity & $15.11(3.29)$ & $16.62(2.51)$ & $<0.001$ & 5.02
\end{tabular}

$S D=$ standard deviation 


\begin{tabular}{|c|c|c|c|c|}
\hline Variable & $\begin{array}{c}\text { Before intervention } \\
\text { Mean (SD) }\end{array}$ & $\begin{array}{c}\text { After intervention } \\
\text { Mean (SD) }\end{array}$ & $P$-value & $t$-value \\
\hline Calcium intake (mg) & $1354.49(581.58)$ & $1473.87(681.42)$ & 0.142 & 1.497 \\
\hline Physical activity (hours/week) & $7.83(3.89)$ & $9.08(4.38)$ & 0.04 & 2.111 \\
\hline
\end{tabular}

$S D=$ standard deviation

(37.5\%), 22 subjects middle (45.8\%) and 8 subjects low $(16.7 \%)$ while these numbers after the intervention were 21 subjects (48.8\%), 18 subjects (41.9\%) and 4 subjects $(9.3 \%)$, respectively. The means of the self-efficacy of calcium intake pre- and post-intervention were 15.11 (SD 3.29) and 16.62 (SD 2.51), respectively; paired $t$-test indicated this difference as significant $(P=0.000)$ (Table 2)

Self-efficacy of physical activity of seven subjects (14.9\%) was good, 13 subjects $(27.7 \%)$ middle and 27 subjects $(57.4 \%)$ low but after the intervention they were figured out as 10 subjects (23.8\%), 21 subjects (50\%) and 11 subjects (26.2\%), respectively. Mean selfefficacy score before the intervention was 18.85 (SD 5.43) and after 21.34 (SD 4.85); paired $t$-test showed this difference to be significant $(P=0.000)$.

The physical activity of 24 subjects before the intervention was severe (34.8\%), 6 subjects had middle (13\%) and 16 had low activity (52\%), however, these scores reached 24 subjects (55.8\%), 12 subjects $(27.9 \%)$ and 7 subjects (16.3\%) after the education. The mean number of hours spent on physical activity per week pre- and post-intervention were 7.83 (SD 3.89) and 9.07 (SD 3.89), respectively, and paired $t$-test showed the difference as significant $(P=0.041)$.

The mean calcium intake of 20 of the subjects was at a desirable level (41.7\%), 22 subjects middle (45.8\%) and 6 subjects low (12.5\%), and after the intervention was 23 subjects (51.1\%), 19 subjects ( $42.2 \%)$ and three subjects $(6.7 \%)$, respectively. The mean of calcium intake in pre- and post-tests was 1354.49 (SD 581.58) and 1473.87 $(\mathrm{SD} 581.58) \mathrm{mg} /$ day, and paired $t$-test showed this difference as insignificant $(P=0.142)($ Table 3$)$.

\section{Discussion}

The significant increase in students' knowledge after the intervention was similar to that found in the results of studies conducted by Nejati et al. among high school students in central Tehran [20], Chan et al. in Hong Kong [21] and Piaseu et al. in Thailand among young nurses [22]. Before the intervention, perceived susceptibility scores of the participants was moderate. Piaseu et al, Hazavehei et al. and Ghaffari et al. [23] suggested that educational intervention increased perceptive susceptibility in young girls. Thus it is prudent to provide the necessary education about osteoporosis to female Iranian adolescents.

In this study, the perceived severity level was moderate before the intervention in more than half of the teenage girls and did not statistically change as shown after it. This finding was consistent with the reports of Tussing and Chapman [24] but different from the findings of Hasavehei et al.

Therefore, it seems we need stronger interventions such as educational films about the side-effects of osteoporosis and perhaps talks by osteoporosis patients.

Perceived benefits of calcium intake and physical activity were high both before and after the intervention, as also reported by Torshizi et al. among postmenopausal woman [25]; however, Piaseu et al., Hazavehei et al., Ghaffari et al and Tussing and Chapman found that intervention significantly increased perceived benefits of calcium intake and physical activity. The difference could have resulted from the fact that in the current study, the level of perceived benefits of the sample was high before intervention.

In this study, the majority of the teenagers were in the group "low perceived calcium intake barriers". However the intervention resulted in a non-significant decrease in barriers $(P=0.187)$; Tussing and Chapman and Zhang [26] reported the same finding as probably due to unhealthy habits and family nutrition. Studies by Ghaffari et al. and Torshizi disagree with the present study which could be explained regarding the different places of the studies and type of the performed intervention.

When woman are interested in increasing their calcium intake, perceived barriers often appear to prevent them from calcium intake [27]. Future research should clarify the barriers to calcium intake and identify approaches to support healthy eating.

Regarding perceived physical activity barriers, most girls belonged to the group with a medium level of barriers while after the intervention they mostly belonged to the group with a low level of barriers. This reduction was statistically significant, indicating that teenage girls could overcome perceived physical activity barriers. The same findings have also been reported by Hasavehei et al, Piaseu et al. and Franko et al. [28]. According to Ziccardi et al, these barriers were possibly belief-based and were reduced by education [29]. Zhang found that education could not change the 
physical activity barriers, though the subjects were young.

Similar to a study published by Baheiraei et al. [30], the results of this study showed that calcium intake self-efficacy was medium in the majority of girls before intervention. After intervention, calcium intake self-efficiency increased to a desirable level and this was statistically meaningful. $(P<0.001)$ This result was similar to the findings of Tussing and Chapman, Piaseu et al., Jung [31], and Chan and Rodsik et al. In contrast to the results of the present study, in Nieto-Vàzquez's study, the educational intervention had no effect on women's self-efficacy.

Before the intervention, teenage girls' physical activity self-efficacy was poor, which reached a middle level after the intervention: a significant increase $(P=0.000)$. A statistically meaningful increase also was shown in physical activity. Hsieh et al. found self-efficacy a better predictor of engaging in osteoporosis preventive behaviour [32]. Results of a study by Gammage et al. suggested the role of physical activity, self-efficacy and health motivation in doing vigorous physical activity among women [33].

Our intervention significantly increased physical activity behaviour hours per week from 7.83 to 9.08 among girls $(P=0.041)$, as also reported by Hazavehei et al. and Nakatani et al. [34]. In contrast to our study's results, the effect of education in Sedlak's study on women did not indicate any significant increase of weight-bearing exercises.
The amount of calcium intake by teenage girls before the intervention was at a medium level which was in accordance with the results of Sharma et al.'s study, yet in Jalili et al.'s study [35]. However, this balanced amount can be due to the modifying factors beside the other perceptions such as financial status, as well as the calcium intake evaluation method used in the present study which was from both milky and non-milky resources.

In our study, calcium intake increased by $119.38 \mathrm{mg}$ after the intervention. Similar to the results of the present study, in Sedlak et al.'s study the educational intervention on three different groups of women did not lead to any significant increase in calcium intake.

In contrast, our findings differed from the results of the studies conducted by Hasavehei et al., Nakatani et al. and Laslett et al. among adults aged $\geq$ 50 years old and Babatunde et al. among older adults [36]. The difference could be due to the difference in evaluation methods of calcium intake or the effect of culture and the age of the participants. However, other behavioural change models can be employed in this regard; for example, in studies conducted by $\mathrm{Lv}$ et al. [37] and Monios et al. [38], theorybased interventions based on theory of planned behaviour effectively increased dietary calcium and vitamin $\mathrm{D}$ intake.

\section{Limitations}

The validity and reliability of the questionnaire used in the present study needs further psychometric evaluation. Figures illustrating consumption of foods containing calcium was based on the participants' self-report, which could be different from their actual food consumption. This fact is also true about their physical activity. The absence of a control group in order to compare to the results of experimental group was another limitation of the study. Due to the short period of the study's duration, determining the effect of the applied intervention in long run is not possible.

\section{Conclusion}

In conclusion, considering the fact that the Health Belief Model had a positive impact on increasing knowledge, perceptions and self-efficacy in teenage girls and improved preventive behaviour to combat osteoporosis, this study could be used as a model for promoting a healthy lifestyle in order to prevent diseases associated with old age. Further studies should have more comprehensive interventions on the structures of calcium intake benefits and barriers and use other behavioural change theories. It is advised that researchers explain social and behavioural barriers in calcium intake in different cultural contexts.

\section{Acknowledgements}

We are grateful to the authorities of the Iranian Ministry of Education and high school teachers for their cooperation.

Funding: This research has been supported by Baqiyatallah University of Medical Sciences.

Competing interests: None declared.

\section{References}

1. Wallace I et al. An evaluation of an enhanced fracture liaison service as the optimal model for secondary prevention of osteoporosis. Journal of the Royal Society of Medicine Short Report, 2011, 2:8.

2. Curtis JR et al. Population-based fracture risk assessment and osteoporosis treatment disparities by race and gender. Journal of General Internal Medicine, 2009, 24:956-962.

3. Schuiling KD, Robinia K, Nye R. Osteoporosis update. Journal of Midwifery \& Women's Health, 2011, 56:615-627.
4. Larijani B et al. Normative data of bone mineral density in healthy population of Tehran, Iran: a cross sectional study. BMC Musculoskeletal Disorders, 2005, 6:38.

5. Salehi I et al. High prevalence of low bone density in young Iranian healthy individuals. Clinical Rheumatology, 2009, 28:173-177.

6. Lowe NM et al. Dietary calcium intake, vitamin D status, and bone health in postmenopausal women in rural Pakistan. Journal of Health, Population, and Nutrition, 2011, 29:465-470. 
7. Tüzün S et al. Turkish Osteoporosis Society. Incidence of hip fracture and prevalence of osteoporosis in Turkey: the FRACTURK study. Osteoporosis International, 2012, 23:949-955.

8. El-Hajj Fuleihan G, Gemma Adib M, Nauroy L. The Middle East \& Africa Regional Audit. Epidemiology, costs \& burden of osteoporosis in 2011. Nyon, Switzerland, International Osteoporosis Foundation, 2011:13-48.

9. Ohta H. [Development and aging of bone in the female life cycle]. Clinical Calcium, 2011, 21:1291-1298 [in Japanese].

10. Theintz $\mathrm{G}$ et al. Longitudinal monitoring of bone mass accumulation in healthy adolescents: evidence for a marked reduction after 16 years of age at the levels of lumbar spine and femoral neck in female subjects. Journal of Clinical Endocrinology and Metabolism, 1992, 75:1060-1065.

11. Babatunde OO, Forsyth JJ, Gidlow CJ. A meta-analysis of brief high-impact exercises for enhancing bone health in premenopausal women. Osteoporosis International, 2012, 23:109-119.

12. Shin S] et al.[Knowledge, health belief, and self-efficacy related to osteoporosis]. Taehan Kanho Hakhoe Chi, 2005, 35:850-857 [in Korean].

13. Moodi $\mathrm{M}$ et al. Evaluation of breast self-examination program using Health Belief Model in female students. Journal of Research in Medical Sciences, 2011, 16:316-322.

14. McLeod KM, Johnson CS. A systematic review of osteoporosis health beliefs in adult men and women. Journal of Osteoporosis, 2011, 2011:197454.

15. Chan MF et al. Evaluation of an osteoporosis prevention education programme for young adults. Journal of Advanced Nursing, 2007, 57:270-285.

16. Turner LW et al. Design and implementation of an osteoporosis prevention program using the Health Belief Model. American Journal of Health Studies, 2004, 19:115-121.

17. Hazavehei SM, Taghdisi MH, Saidi M. Application of the Health Belief Model for osteoporosis prevention among middle school girl students, Garmsar, Iran. Education for Health (Abingdon), 2007, 20:23.

18. Strong WB et al. Evidence based physical activity for school-age youth. Journal of Pediatrics, 2005, 146:732-737.

19. Musaiger AO. Food Composition Tables for Kingdom of Bahrain Manama, University of Bahrain, Arab Center for Nutrition, 2011:34-60.

20. Nejati S, Rasoulzadeh N, Sedighiyani A. [The effectiveness of education on prevention of osteoporosis among high schoo female students]. Hayat Journal of Faculty of Nursing \& Midwifery, 2009, 15:59-65 [In Farsi].

21. Chan MF et al. Evaluation of an osteoporosis prevention education programme for young adults. Journal of Advanced Nursing, 2007, 57:270-285.

22. Piaseu N, Belza B, Mitchell P. Testing the effectiveness of an osteoporosis educational program for nursing students in Thailand. Arthritis and Rheumatism, 2001, 45:246-251.

23. Ghaffari $M$ et al. Effect of health belief model-based intervention on promoting preventive behaviors about osteoporosis among students of female middle schools in Isfahan, Iran. Journal of Education and Health Promotion $\_$2012, 1:14.

24. Tussing L, Chapman-Novakofski K. Osteoporosis prevention education: behavior theories and calcium intake. Journal of the American Dietetic Association, 2005, 105:92-97.

25. Torshizi L et al. The effect of education-based on the Health Belief Model on preventive factors of osteoporosis among postmenopausal women. Iran Journal of Nursing, 2009, 22:71-82.

26. Zhang YP et al. Evaluation of educational program on osteoporosis awareness and prevention among nurse students in China. Nursing \& Health Sciences, 2012, 14:74-80.

27. Blalock SJ. Toward a better understanding of calcium intake: behavioral change perspectives. Journal of Reproductive Medicine, 2005, 50(Suppl.):901-906

28. Franko DL et al. Motivation, self-efficacy, physical activity and nutrition in college students: randomized controlled trial of an internet-based education program. Preventive Medicine, 2008, 47:369-377.

29. Ziccardi SL, Sedlak CA, Doheny MO. Knowledge and health beliefs of osteoporosis in college nursing students. Orthopedic Nursing, 2004, 23:128-133.

30. Baheiraei A et al. Psychometric properties of the Persian version of the osteoporosis knowledge and health belief questionnaires. Maturitas, 2005, 50:134-139.

31. Jung ME et al. Increasing calcium intake in young women through gain-framed, targeted messages: a randomised controlled trial. Psychology \& Health, 2011, 26:531-547.

32. Hsieh $\mathrm{CH}$ et al. Factors influencing osteoporosis preventive behaviours: testing a path model. Journal of Advanced Nursing, 2008, 62:336-345.

33. Gammage KL et al. Gender differences in osteoporosis health beliefs and knowledge and their relation to vigorous physical activity in university students. Journal of American College Health, 2012, 60:58-64.

34. Nakatani Y et al. Effect of distributing an evidence-based guideline for prevention of osteoporosis on health education programs in municipal health centers: a randomized controlled trial. Journal of Epidemiology, 2012, 22:103-112.

35. Jalili $Z$ et al. Knowledge, attitude and preventive practice of women concerning osteoporosis. Iranian Journal of Public Health, 2007, 36:19-25.

36. Laslett LL et al. Osteoporosis education improves osteoporosis knowledge and dietary calcium: comparison of a 4 week and a one-session education course. International Journal of Rheumatic Diseases, 2011, 14:239-247.

37. Lv N, Brown JL. Impact of a nutrition education program to increase intake of calcium-rich foods by Chinese-American women. Journal of the American Dietetic Association, 2011, 111:143-149.

38. Manios $\mathrm{Y}$ et al. Changes in diet quality score, macro- and micronutrients intake following a nutrition education intervention in postmenopausal women. Journal of Human Nutrition and Dietetics, 2007, 20:126-131. 